\title{
Frequency Bandwidth Management of Satellite Communication Network
}

\author{
Zhengqun $\mathrm{Hu}^{1}$, a \\ ${ }^{1}$ National Astronomical Observatory, Chinese Academy of Sciences, Beijing, China \\ ahuzq@nao.cas.cn
}

Keywords: Satellite communication; Bandwidth; Network; Management

\begin{abstract}
Satellite communication network has a wide application prospect based on its unique advantages. Satellite frequency bandwidth is an important component of satellite communication network. However satellite bandwidth resources are high cost and relatively limited, it is essential to optimally use frequency band resource. In order to design a certain satellite communication network, taking into account the satellite communication network characteristic with dynamic network topology and satellite bandwidth resources restriction, according to the business type of system requirements, we design and allocate frequency bandwidth in this the satellite communication network, illustrate the management model of frequency bandwidth and allocation principle.
\end{abstract}

\section{Introduction}

Satellite communication has characteristics of wide coverage and long distance communication [1], flexible multiple access mode, etc., which has an extensive application value. Satellite bandwidth is blood of satellite communication [2, 3], which determines the capacity of satellite communication. On the one hand, it is high cost to launch satellites. There is limited frequency bandwidth on one satellite. On the other hand, the orbital resource is limited, it is not impossible to launch many satellites, which can form frequency interference. Satellite bandwidth has always been the bottleneck of satellite communication development. TDMA communication system is usually used in return link direction in satellite communication. Satellite bandwidth design, the effective management of bandwidth resources and the principle of frequency band resource allocation is powerful guarantee of satellite communication system in optimization operation.

\section{Frequency Bandwidth Design}

Satellite frequency bandwidth design is key technology in TDMA satellite communication network. We should consider user capacity with each TDMA frequency bandwidth in the design of carrier frequency bandwidth. If TDMA frequency bandwidth is too large, although a large frequency bandwidth can accommodate large numbers of user, the terminal needs to configure large power amplifier and satellite antenna. Else TDMA frequency bandwidth is too small, we need to configure more carriers in the whole network, frequently TDMA frequency hopping also bring additional overhead, which is not conducive for system management. Therefore, reasonable frequency bandwidth design is important to balance the numbers of carrier and terminal size $[4,5]$.

Bandwidth of Video Transmission. Due to the real-time transmission requirements, the demand to frequency bandwidth is the maximum in. The frequency bandwidth is designed according to system demand. For a single satellite remote video transmission station, the amount of video data = resolution $\times$ frames $\times$ digit code $\times$ color, frame rate is 25 , digit code is 8 , the color value is 2 , according to the compression ratio value separately by $30 / 60$, we can calculate information rate, modulation and required symbol rate in video transmission which is shown in Table 1 and Table 2. The relationship between resolution and required frequency bandwidth is shown in Fig. 1. 
Table 1 Relationship of Resolution, Information Rate, Modulation Mode and Required Symbol Rate (Compression: 30)

\begin{tabular}{|c|c|c|c|}
\hline $\begin{array}{c}\text { Resolution } \\
\text { [Pixel] }\end{array}$ & $\begin{array}{c}\text { Information } \\
\text { Rate [Mbps] }\end{array}$ & $\begin{array}{c}\text { Modulation } \\
\text { Mode }\end{array}$ & $\begin{array}{c}\text { Symbol Rate } \\
{[\mathrm{M} \text { Symbol/s] }}\end{array}$ \\
\hline \multirow{3}{*}{$1920 * 1080$} & \multirow{3}{*}{27.648} & BPSK & 40.070 \\
\cline { 3 - 4 } & & QPSK & 20.035 \\
\cline { 3 - 4 } & & 8PSK & 13.357 \\
\hline \multirow{3}{*}{$960 * 720$} & \multirow{3}{*}{9.216} & BPSK & 13.356 \\
\cline { 3 - 4 } & & QPSK & 6.678 \\
\cline { 3 - 4 } & & 8PSK & 4.452 \\
\hline \multirow{3}{*}{$640 * 480$} & \multirow{3}{*}{4.096} & BPSK & 5.936 \\
\hline \multirow{3}{*}{$320 * 240$} & & QPSK & 2.968 \\
\cline { 3 - 4 } & \multirow{3}{*}{1.024} & 8PSK & 1.979 \\
\hline \multirow{3}{*}{$160 * 120$} & & BPSK & 1.484 \\
\cline { 3 - 4 } & \multirow{3}{*}{0.256} & QPSK & 0.742 \\
\cline { 3 - 4 } & & 8PSK & 0.495 \\
\cline { 3 - 4 } & & BPSK & 0.372 \\
\cline { 3 - 4 } & & QPSK & 0.186 \\
\hline
\end{tabular}

Table 2 Relationship of Resolution, Information Rate, Modulation Mode and Required Symbol Rate (Compression: 60)

\begin{tabular}{|c|c|c|c|}
\hline $\begin{array}{l}\text { Resolution } \\
\text { [Pixel] }\end{array}$ & $\begin{array}{l}\text { Information } \\
\text { Rate [Mbps] }\end{array}$ & $\begin{array}{l}\text { Modulation } \\
\text { Mode }\end{array}$ & $\begin{array}{l}\text { Symbol Rate } \\
\text { [M Symbol/s] }\end{array}$ \\
\hline \multirow{3}{*}{$1920 * 1080$} & \multirow{3}{*}{13.824} & BPSK & 20.035 \\
\hline & & QPSK & 10.017 \\
\hline & & 8PSK & 6.678 \\
\hline \multirow{3}{*}{$960 * 720$} & \multirow{3}{*}{4.608} & BPSK & 6.678 \\
\hline & & QPSK & 3.339 \\
\hline & & 8PSK & 2.226 \\
\hline \multirow{3}{*}{$640 * 480$} & \multirow{3}{*}{2.048} & BPSK & 2.968 \\
\hline & & QPSK & 1.484 \\
\hline & & $8 \mathrm{PSK}$ & 0.989 \\
\hline \multirow{3}{*}{$320 * 240$} & \multirow{3}{*}{0.512} & BPSK & 0.742 \\
\hline & & QPSK & 0.371 \\
\hline & & 8PSK & 0.247 \\
\hline \multirow{3}{*}{$160 * 120$} & \multirow{3}{*}{0.128} & BPSK & 0.186 \\
\hline & & QPSK & 0.093 \\
\hline & & 8PSK & 0.062 \\
\hline
\end{tabular}




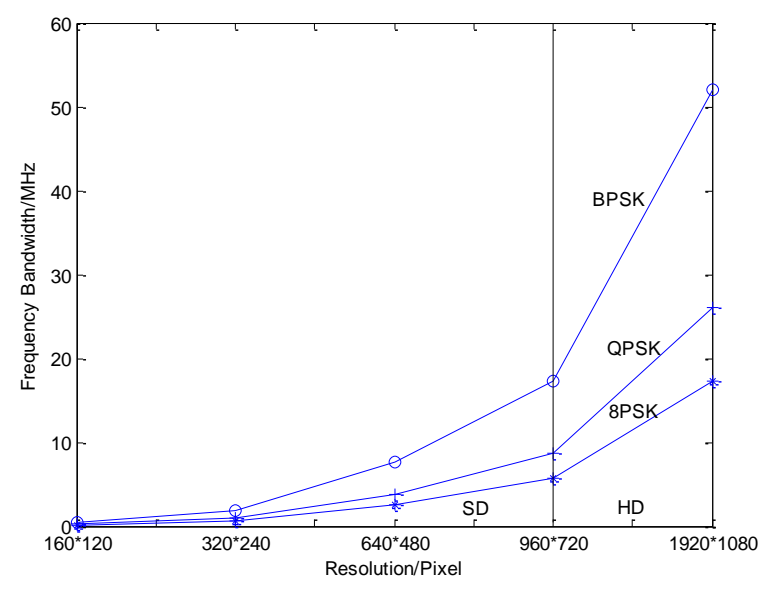

a

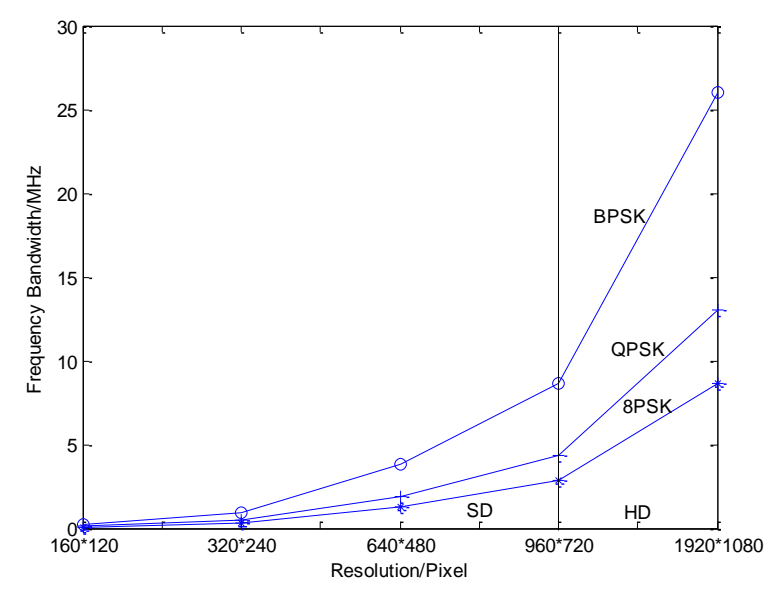

b

Figure 1. Relationship between resolution and required frequency bandwidth

(a. Compression rate $=30 ; \mathrm{b}$. Compression rate $=60$ )

Comprehensively considering video quality and system resource requirements, channel quality and modulation mode, we can select each satellite remote station backhaul video resolution of $640 * 480$, modulation mode is $8 \mathrm{PSK}$, frequency bandwidth is $1.3846 \mathrm{MHz}$. If we determine the concurrent rate of remote station, the total required frequency bandwidth for the video transmission system will be determined.

Bandwidth of Satellite Telephone. The bandwidth of VOIP phone in each remote station is related to the number to-satellite signal and the duration of call. VOIP phone bandwidth will be determined by the voice encoding, Baotou compression rate and load size. For example, the use of G.729 algorithm [6], the payload is 40Bytes, no Baotou compression, a VOIP phone about occupies $16 \mathrm{kbps}$ bandwidth, if Baotou is compression, a VOIP phone about occupies 10kbps bandwidth.

The relay circuit utilization rate is designed as $0.7 \mathrm{Erl}$, busy time concentration factor is $10 \%$, the average call takes up 180 seconds, a single occurrence amount in the busiest time is as follows:

$>$ The most busy then a single occurrence amount $=$ numbers of relay circuit $\times$ circuit utilization rate $\times 3600$ / average occupation time

$>$ The data acquisition rate $=$ busy time singular volume $\times 200 \times 8 / 0.5 /(0.5 \times 3600)$

$>$ Storage capacity in a single day traffic $=$ singular volume $\times 200 \times 2 \times 30 \times(1+0.25 * 2) /$ 1024 / 1024 / 1024

If we determine the concurrent rate of remote station, the total required frequency bandwidth for the satellite VOIP phone will be determined.

\section{Frequency Bandwidth Management}

Although the processing ability of satellite is very powerful in satellite communication network system, the satellite still can't afford the ability to allocate user bandwidth [7]. Satellite bandwidth only can be allocation and management in satellite ground hubs station. Each satellite remote station monitors the transmitted signal from hubs station, at the same time which is received by hubs station in order to adjust the transmission carrier frequency, so that each remote station can correctly receive the satellite hubs station signal. The network control center reads the corresponding satellite remote station signal receiving frequency from the demodulator of hubs station in timing cycle, in order to determine whether it is within the allowable range and judge whether need to be step adjustment. The orders of step adjustment will be packed in network management command which is transmitted to corresponding satellite remote station to control frequency. Fig. 2 shows the structure model of bandwidth resource management system. 


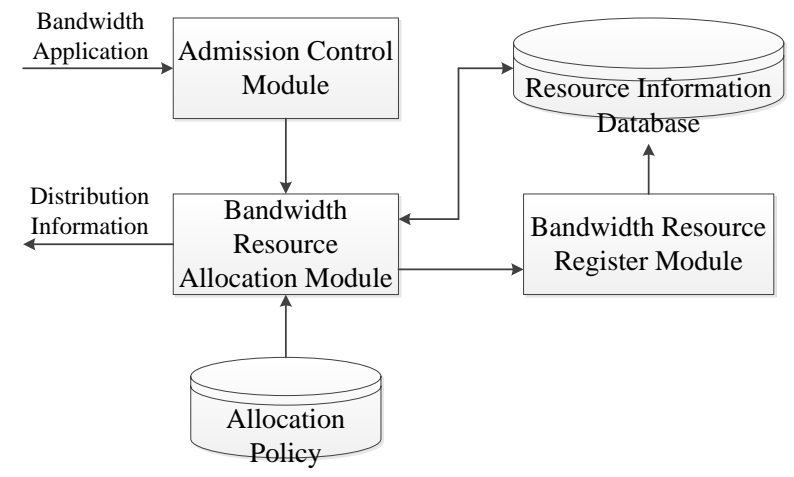

Figure 2. Structure model of bandwidth resource management system

Satellite remote station need to apply in resource management system to utilize the bandwidth [8], which is responsible for processing satellite remote station bandwidth request, the admission control module is responsible for acceptance or rejection of satellite remote application request, bandwidth resource allocation module is responsible for the allocation of bandwidth resources according to the resource allocation strategy and timely amend the information resources in the library. Bandwidth resource register module is responsible for dynamic collection of satellite dynamic bandwidth resource information and timely manner the collection of bandwidth resources information into the resource information database, the new bandwidth resources statistics as shown in Table 3.

Table 3 Bandwidth Resource Information

\begin{tabular}{l}
\hline Brief description \\
\hline Link numbers \\
Tables to describe every link bandwidth \\
information \\
Entries of containing bandwidth information \\
Distinguish the link bandwidth \\
Link number \\
Satellite number \\
Resource type \\
Total bandwidth \\
Usage amount of bandwidth \\
Residual amount of bandwidth \\
Minimum allocation unit \\
\hline
\end{tabular}

Bandwidth resource management system not only manages the use process of bandwidth resources, but also manages the entire life cycle of bandwidth resources. The life cycle of bandwidth resource includes the whole process of registration, allocation and cancellation.

\section{Frequency Bandwidth Allocation Criterion}

Fairness is an important criterion for frequency bandwidth allocation. Different fairness criterions can be integrated and complementary with each other, forming new fairness criterion. A weighted Max-Min fairness criterion is integrated with Max-Min fairness criterion and weighted fairness criteria. The proportional fairness $(p, a)$ is proposed in reference [9], which is unified by Max-Min fairness and weighted fairness. The utility function [10] is shown in Eq.1 which realizes different fairness criteria. 


$$
U_{i}\left(x_{i}, a\right)=\left\{\begin{array}{cc}
(1-a)^{-1} x_{i}^{1-a} & a \neq 1 \\
\lg x_{i} & a=1
\end{array}\right.
$$

Where $a \geq 0$, different value corresponds to different fairness criteria. The corresponding allocation criterion is to maximize the throughput when $a=0$; the corresponding proportional fairness when $a=1$; the corresponding Max-Min fairness criterion when $a=\infty$.

The comprehensive fairness criterion can not only solve the more demand of bandwidth resources in some remote stations allocation, but also improve the overall throughput of the whole network.

\section{Conclusions}

With the importance and complexity of satellite communication networks, bandwidth resource management is very important for a satellite system. Aim at the satellite communication network characteristic with dynamic network topology and satellite bandwidth resources restriction, according to the business type of system requirements including video transmission and satellite phone. We design and allocate frequency bandwidth in this the satellite communication network, illustrate the management model of frequency bandwidth and bandwidth allocation principle which is operating with better performance. It has a certain reference value in relevant technical field.

\section{Acknowledgements}

The work was supported by the National Natural Science Foundation of China (11573041, 11473045, 61271284); the Young Researcher Grant of National Astronomical Observatories 2015, Chinese Academy of Sciences (Grant No.O835032002); the Key Research Program of the Chinese Academy of Sciences (KGFZD-125-14-005-2); Development Program (863 program) Project of China (Grant No.2012AA120800); the Pilot Program for New and Interdisciplinary Subjects of Chinese Academy of Sciences (KJCX2-EW-J01) and the Knowledge Innovation Program of Chinese Academy of Sciences (KGCX2-EW-4071).

\section{References}

[1] S.Q Wu: Introduction to Satellite Communications (Publishing House of Electronics Industry, China 2007).

[2] G. L. Liu: Satellite Communication Network Technology (Posts and Telecom Press, China 2015).

[3] Q. Guo: Satellite Communication System (Publishing House of Electronics Industry, China 2010).

[4] N. Xu, X. K. Hao and Z. Xu: Radio Communications Technology, Vol.38(2012)No.2, p.45.

[5] L. M. Chen: Research on radio resource management method for integration network of satellite and ground (Ph.D., Harbin Institute of Technology, China 2014), p.34.

[6] Y.F. Hu: IET Commun, Vol. 13 (2010) No.4, p.1519.

[7] E. Halvorson: Proc. the 33rd IEEE Sarnoff Symposium (Princeton, US, 2010). Vol.1, p.1.

[8] Y. Qin, J. Zhang and T. Zhang: Computer Science, Vol. 37 (2010) No.2, p.23.

[9] F.P. Kelly: European Transactions on Telecommunication, Vol. 8 (1997) No.1, p.33.

[10] J. Mo and J. Walrand: IEEE/ACM Transaction on Networking, Vol. 8 (2000) No.5, p.550. 